\title{
Perfil do consumo de álcool e prática do beber pesado episódico entre universitários brasileiros da área da saúde
}

\author{
Alcohol consumption and episodic heavy drinking among \\ undergraduate students from the health area of a Brazilian university
}

Marina Pelicioli', Cristiane Barelli², Carla Beatrice Crivellaro Gonçalves', Siomara Regina Hahn³, José lvo Scherer

\section{RESUMO}

Objetivo: Identificar entre os universitários da área da saúde de uma universidade brasileira a prevalência, o perfil do consumo de álcool e a prática do beber pesado episódico (BPE). Métodos: Estudo transversal, quantitativo, com amostra randomizada dos universitários dos 12 cursos da saúde. Foram aplicados questionários individualizados, em formulário eletrônico, utilizando a escala AUDIT, após consentimento informado. Foi realizada análise estatística descritiva e inferencial. Resultados: Entre os 619 estudantes selecionados, a prevalência de consumo de álcool foi de $85 \%$, com perfil do consumo de baixo risco (77,1\%). Consumo nocivo e provável dependência foram detectados em 2,7\%. A prática do BPE foi de 51,6\%, quase o dobro

\section{Palavras-chave}

Consumo de álcool, alcoolismo, beber pesado episódico, estudantes universitários.

\section{Keywords}

Alcohol consumption, alcoholism, heavy drinking episodic, university students. em comparação com outros estudos. As características que mais se associaram ao consumo de álcool foram: ser solteiro, do sexo masculino e frequentar festas semanalmente. Conclusão: $\mathrm{O}$ consumo de álcool é elevado entre os estudantes avaliados, embora a maioria esteja no perfil de baixo risco. Contudo, fica evidente a necessidade de ações educativas para a promoção da saúde do estudante, visando à redução e ao consumo consciente de bebidas alcoólicas.

\section{ABSTRACT}

Objective: To identify among university students of the health area of a Brazilian university the prevalence, alcohol consumption profile and the practice of episodic heavy drinking (EHD). Methods: A cross-sectional, quantitative study with a randomized sample of university students from 12 health courses. Individual questionnaires were used, in an electronic form, using the AUDIT scale, after informed consent. Descriptive and inferential statistical analysis were performed. Results: Among the 619 students selected, the prevalence of alcohol consumption was $85 \%$, profile of low risk consumption (77.1\%). Harmful consumption and probable dependence were detected in $2.7 \%$. The HDE practice was $51.6 \%$, almost double when compared to other studies. The characteristics that were most associated with alcohol consumption were: being single, male and attending parties weekly. Conclusion: Alcohol consumption is high among the students evaluated, although most are in the low risk profile. However, it is evident the need for educational actions to promote student health, aiming at the reduction and conscious consumption of alcoholic beverages.

1 Universidade Federal da Fronteira Sul (UFFS), Saúde da Família e Comunidade. 2 Universidade de Passo Fundo (UPF), Faculdade de Medicina.

3 Universidade de Passo Fundo (UPF), Instituto de Ciências Biológicas.

Endereço para correspondência: Marina Pelicioli Rua João Lângaro, 183, Bairro Planaltina 99062-060 - Passo Fundo, RS, Brasil E-mail:m.pelicioli@gmail.com 


\section{INTRODUÇÃO}

O abuso e a dependência de substâncias químicas, tais como o álcool, ameaçam valores políticos, econômicos e sociais, sendo um indicador negativo de desenvolvimento. Além de onerar gradativamente os gastos com tratamento e internação hospitalar, elevam os índices de acidentes de trânsito, de violência urbana, de anos de vida potencialmente perdidos e de mortes prematuras ${ }^{1-3}$.

A cada ano, cerca de 2 bilhões de pessoas consomem bebidas alcoólicas, o que corresponde a aproximadamente $40 \%$ da população mundial, ou seja, duas pessoas em cada cinco, acima de 15 anos, consomem álcool. Estima-se que anualmente morrem 2 a 2,5 milhões de pessoas devido ao uso de álcool em situações de intoxicações agudas, cirrose hepática induzida pelo álcool, violência e colisões de automóveis ${ }^{4-6}$.

O conceito de binge drinking ou beber pesado episódico (BPE) vem sendo utilizado para definir a conduta dos jovens em relação ao consumo de álcool. Corresponde ao consumo em quantidade excessiva de álcool em um determinado episódio, quando há o consumo de cinco ou mais doses de bebidas alcoólicas em um único espaço de tempo, independentemente da frequência de consumo $0^{7-9}$.

Logo, o consumo do álcool, bem como a prática do BPE, representam um grave problema de saúde pública, sendo um dos agravos não transmissíveis mais relevantes da atualidade. Aqui entendemos agravos não transmissíveis como situações caracterizadas por múltiplas causas ou longo tempo de evolução, ou acontecimentos pontuais (acidentes, violência, acidente vascular cerebral), com evolução para óbito ou recuperação com sequelas e necessidade de reabilitação e readaptação às novas condições de vida. As doenças e agravos não transmissíveis estão diretamente relacionados à forma como a sociedade está organizada e ao estilo de vida da população ${ }^{10}$.

Quando os jovens ingressam na universidade, muitas vezes eles se afastam do seu círculo de relacionamentos familiares e sociais, o que pode desencadear situações de crise e busca de estratégias para enfrentar essa situação. A literatura científica tem demonstrado que os estudantes universitários são mais vulneráveis ao consumo de bebidas alcoólicas, pela oferta demasiada de festas e locais de lazer com venda livre de bebidas alcoólicas. Um estudo realizado em São Paulo, Brasil, com estudantes da área da saúde, evidenciou que quanto maior a frequência em festas, maior o consumo de bebidas alcoólicas $2,3,11$.

A adaptação ao ambiente universitário representa uma mudança na rotina do estudante, pois envolve metodologia diferente das vivenciadas no ensino secundário. Ainda, deve-se considerar que muitas vezes há o despreparo por parte dos professores, a extrema dedicação imposta pelos cursos da área da saúde, a quantidade excessiva de conteúdos, nem sempre contextualizados e integrados, e a aplicação de práticas avaliativas baseadas na memorização. Tudo isso pode representar agentes estressores e desencadeantes na busca de enfrentamento por meio do consumo de álcool, por vezes caracterizado por momentos de integração entre a população estudanti|6,11.

$\mathrm{Na}$ atualidade, vivenciamos uma situação em que o uso de álcool e drogas pela população jovem tem sido um tema de ampla importância e representa, de certa forma, um problema social complexo, pois os órgãos governamentais e não governamentais não têm conseguido gerar respostas efetivas para o problema. Essa situação pode ser ampla à sociedade em geral, na medida em que a família e outras instituições tais como igrejas, escolas e universidades vêm enfrentando dificuldades para reagir em relação a ocorrências vinculadas ao uso de álcool e drogas ${ }^{3,12}$.

O objetivo deste estudo foi identificar, entre universitários brasileiros de 12 cursos da área da saúde, a prevalência e o perfil do consumo de álcool, além de determinar a frequência do BPE, bem como os aspectos que mais se associam a esses comportamentos.

\section{MÉTODOS}

O protocolo de pesquisa foi aprovado pelo Comitê de Ética em Pesquisa da Universidade de Passo Fundo (Parecer no 327917/2013). Trata-se de um estudo transversal, quantitativo, prospectivo, realizado com os estudantes dos 12 cursos de graduação da área da saúde de uma instituição de ensino superior do Rio Grande do Sul, Brasil. A instituição se situa na região norte do estado do Rio Grande do Sul e sua natureza jurídica é privada, filantrópica e caracterizada como uma universidade comunitária, conforme os termos do artigo 213 da Constituição Federal do Brasil.

A população do estudo foi composta por estudantes regularmente matriculados nos 12 cursos de graduação da área da saúde, no campus central, do período diurno e modalidades de formação profissional ou bacharelado, a saber: Medicina, Enfermagem, Farmácia, Fisioterapia, Ciências Biológicas, Serviço Social, Odontologia, Educação Física, Medicina Veterinária, Fonoaudiologia, Psicologia e Nutrição. Foram excluídos alunos menores de 18 anos, alunos de cursos de graduação na modalidade licenciatura e estudantes em tratamento para dependência química.

O cálculo do tamanho amostral foi realizado pela fórmula de Cochran ${ }^{13}$, com prevalência esperada de consumo de álcool de 50\%, erro amostral de 5\% e intervalo de confiança de 95\%. A amostra calculada foi de 1.877 alunos, incluindo $20 \%$ de perdas e recusas, levando em consideração o número total de alunos matriculados, equiparados por níveis em andamento no curso durante o segundo semestre letivo de 2013. A randomização foi feita conforme o cálculo amostral, por 
meio de planilhas no programa Microsoft Excel, utilizando números aleatórios que remetiam aos alunos enumerados nas listas de chamadas de cada turma.

Os sujeitos randomizados primeiramente foram convidados a participar da pesquisa por e-mail, ocasião em que os pesquisadores explicavam os propósitos do estudo e orientavam para o acesso ao questionário. A coleta de dados ocorreu por meio de questionários de autopreenchimento, individualizados, em formato de formulários eletrônicos, elaborados na plataforma de acesso livre Google Docs.

Foi enviado aos participantes do estudo, por e-mail, o link que remetia ao questionário. O primeiro item do questionário eletrônico era o Termo de Consentimento Livre e Esclarecido, sendo uma questão obrigatória, pois, sem o consentimento, o questionário não seria submetido.

O instrumento de coleta de dados foi composto por 37 questões e, ao término destas, havia um campo para registro de comentários. Na primeira parte, o respondente informava suas características pessoais: sexo, idade, curso, nível em que estava matriculado, raça, religião, moradia e relacionamentos interpessoais. Na sequência, foram incluídas as questões do AUDIT (Alcohol Use Disorders Identification Test), um questionário validado internacionalmente, preconizado pela Organização Mundial da Saúde como instrumento de rastreamento para verificar os padrões de consumo de álcool $^{14-16}$. O AUDIT é composto por 10 perguntas fechadas e, de acordo com a pontuação obtida, auxilia na identificação de quatro diferentes padrões de consumo: uso de baixo risco ou zona de risco I (0 a 7 pontos); uso de risco ou zona de risco II (8 a 15 pontos); uso nocivo ou zona de risco III (16 a 19 pontos); e provável dependência ou zona de risco IV (20 ou mais pontos). O critério para interpretação do BPE foi uma resposta diferente de "nunca" para a questão 3 do AUDIT ("Qual a frequência com que você consome cinco ou mais doses de bebida alcoólica em uma ocasião?") ${ }^{7-9}$.

Os dois desfechos principais foram o consumo de álcool e o BPE, e as variáveis associadas foram: opção religiosa, dedicação à escolha religiosa, relacionamentos interpessoais, onde e com quem mora, momento em que se encontra no curso e frequência em festas e de encontros com amigos.

Foram realizadas análises para os 12 cursos no geral e análises individualizadas para os cursos de Farmácia, Medicina e Enfermagem, pois apresentaram o maior número de respondentes.

Para análise dos resultados, os dados foram codificados e analisados por estatística descritiva e inferencial. Para análise das variáveis categóricas, foi utilizado o teste de qui-quadrado. As comparações foram realizadas por meio do teste $t$ de Student, e as associações foram calculadas pelo coeficiente de correlação de Pearson, considerando significativos valores de $\mathrm{p}$ inferiores a 0,05 .

\section{RESULTADOS}

A amostra investigada abrangeu 619 estudantes de 12 cursos da área da saúde. Observamos o predomínio de mulheres (82,9\%), e a idade variou de 18 a 34 anos. Quanto aos relacionamentos interpessoais, a maioria referiu estar namorando $(46,5 \%)$ ou ser solteiros $(45,3 \%)$. A fonte de renda mais prevalente foram os subsídios dos pais, seguida de emprego formal ou informal e estágio remunerado.

A prevalência do consumo do álcool foi avaliada no geral, ou seja, o consumo de álcool para os 12 cursos da área da saúde, e também foram comparados os três cursos que atenderam $50 \%$ ou mais da meta de respondentes previstos pelo cálculo amostral, a saber: Farmácia, Medicina e Enfermagem.

A prevalência do consumo de álcool foi elevada (85\%) e semelhante entre os três cursos. Em relação ao BPE, os 12 cursos apresentaram uma prática de $51,6 \%$, e os cursos de Farmácia e Medicina alcançaram índices superiores ao desempenho dos cursos no geral (Tabela 1).

Tabela 1. Prevalência do consumo de álcool e prática do beber pesado episódico (BPE) para os universitários brasileiros dos 12 cursos e comparação entre os cursos de maior número de respondentes (Farmácia, Medicina e Enfermagem)

\begin{tabular}{lcc}
\hline \multirow{2}{*}{ Curso de graduação } & \multicolumn{2}{c}{ Prevalência (\%) } \\
\cline { 2 - 3 } & Consumo deálcool* & BPE** $^{*}$ \\
\hline Farmácia $(n=58)$ & 94,6 & 64,3 \\
Medicina $(n=111)$ & 93,7 & 68,5 \\
Enfermagem $(n=70)$ & 74,3 & 42,9 \\
Todos os cursos $(n=619)$ & 85,0 & 56,1 \\
\hline
\end{tabular}

* 0 não consumo de álcool foi identificado quando a resposta à questão 1 do AUDIT ("Com que frequência você consome bebidas alcoólicas?) era NUNCA.

** BPE foi identificado quando a resposta à questão 3 do AUDIT ("Qual a frequência com que você consome cinco ou mais doses de bebida alcoólica em uma ocasiäo?") era diferente de NUNCA.

Em relação ao sexo, os homens apresentaram ocorrência de BPE mais elevada (70,8\%) em comparação com as mulheres $(47,6 \%)$. O momento em que os estudantes se encontravam no curso não foi relevante no comportamento do BPE, e a distribuição entre os semestres do curso foi homogênea $(p=0,709)$. Os estudantes que praticavam o BPE moravam na mesma cidade em que o curso estava instalado $(p<0,006)$.

A frequência de consumo de álcool entre os respondentes variou de duas a quatro vezes ao mês $(46,9 \%)$ a mensal (40\%). O padrão de consumo de álcool de baixo risco e de risco foram os comportamentos predominantes na amostra (Figura 1, Tabela 2).

Quanto ao tipo de bebida ingerida, houve predomínio de fermentados $(41,1 \%)$ em relação a destilados $(23,2 \%)$. Embora o curso de Farmácia tenha apresentado maior prevalência de consumo de bebidas alcoólicas dentre os três cursos, foi o curso que apresentou menos alunos com comportamento 


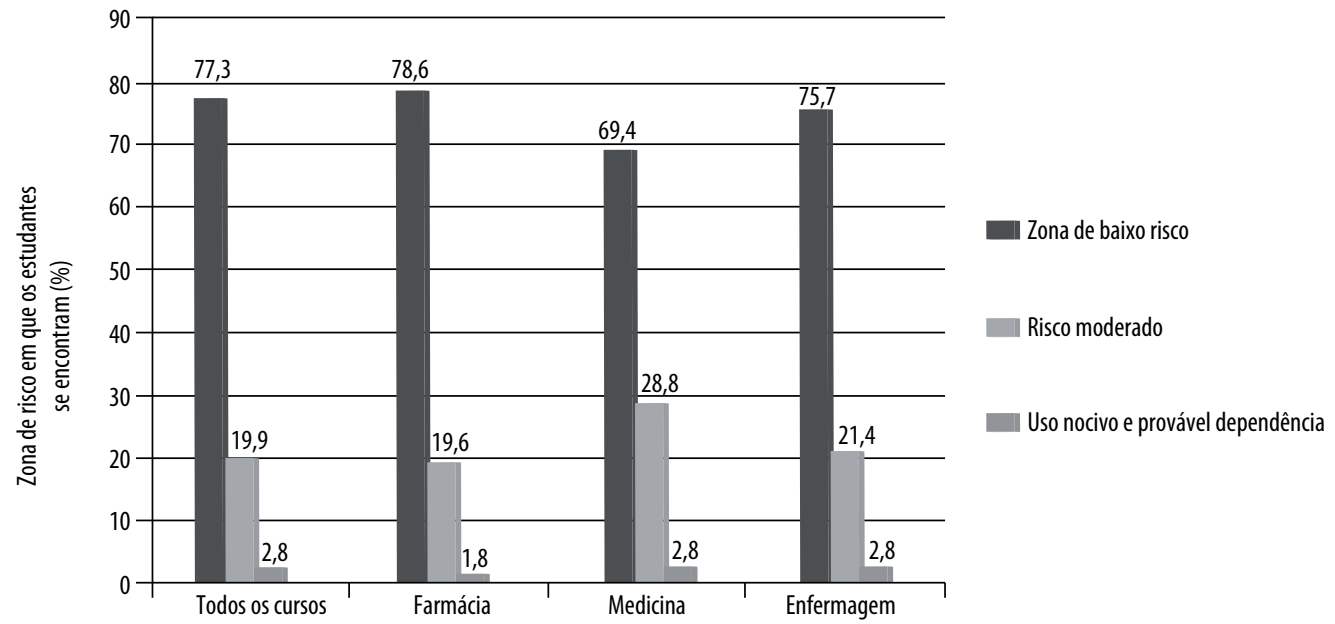

Figura 1. Distribuição e comparação da frequência relativa de perfis gerados pelo questionário AUDIT para os universitários brasileiros dos cursos da área da saúde, bem como a classificação das zonas de risco relativas ao consumo de álcool.

Nota: os perfis de uso nocivo e provável dependência foram agrupados em categoria única.

Tabela 2. Perfil do consumo de bebidas alcoólicas para os universitários brasileiros dos 12 cursos da área da saúde conforme O AUDIT $(n=619)$.

\begin{tabular}{lcc}
\hline Perfil do consum $0^{*}$ & Frequência absoluta $(\mathbf{n})$ & Frequência relativa $(\%)$ \\
\hline Baixo risco & 478 & 77,3 \\
Risco moderado & 124 & 19,9 \\
Nocivo & 10 & 1,7 \\
Provável dependência & 7 & 1,1 \\
\hline
\end{tabular}

* Obtido a partir da pontuação gerada do AUDIT. Uso de baixo risco ou zona de risco I ( 0 a 7 pontos); uso de risco ou zona de risco II (8 a 15 pontos); uso nocivo ou zona de risco III (16 a 19 pontos); e provável dependência ou zona de risco IV (20 ou mais pontos).

de risco em relação ao consumo de álcool. Em contrapartida, o curso de Medicina foi o que teve mais estudantes com comportamento de risco em relação ao consumo de bebidas alcoólicas.

$\mathrm{Na}$ análise geral dos 12 cursos, os fatores que se associaram de forma significativa a maiores escores obtidos no perfil do consumo decorrente da escala AUDIT foram: residir na cidade-sede do curso, não utilizar medicamentos de forma crônica, ser solteiro e frequentar festas uma ou mais vezes por semana. A prática religiosa semanal, independentemente da religião, foi um fator que ocasionou escores no AUDIT e prática de BPE significativamente menores, em comparação com estudantes que praticavam eventualmente ou não seguiam uma opção religiosa $(p<0,005)$. Para a amostra analisada, não ter nenhum relacionamento, ou seja, ser solteiro, proporcionou maior chance de praticar o BPE $(p<0,005)$. Em relação a ir a festas com oferta de bebidas alcoólicas, 92 estudantes que praticavam BPE iam a festas com frequência semanal, ao contrário dos 224 estudantes que não praticam $B P E$, que frequentam festas esporadicamente $(p<0,005)$.

Para os estudantes do curso de Farmácia, os escores do AUDIT foram significativamente maiores entre os que não re- sidiam com os pais/familiares e quando frequentavam festas uma ou mais vezes por semana $(p<0,005)$.

Os estudantes de Enfermagem que apresentaram escores maiores para o AUDIT foram os solteiros $(p=0,032)$ e aqueles que frequentavam festas com periodicidade superior à semanal $(p=0,005)$. Para os estudantes de Medicina, os escores maiores foram obtidos entre os solteiros e aqueles que frequentavam festas uma ou mais vezes por semana ( $p$ $<0,005)$.

\section{DISCUSSÃO}

Os achados desta pesquisa mostram prevalência de consumo de bebidas alcoólicas de $85 \%$ e prática do BPE de $56,1 \%$. Foi predominante o perfil de consumo de baixo risco $(77,1 \%)$ e a prática mais acentuada em indivíduos do sexo masculino, comparados a indivíduos do sexo feminino. De acordo com o I Levantamento Nacional sobre o Uso de Álcool, Tabaco e Outras Drogas, realizado em 2010, entre universitários das 27 capitais brasileiras, 86,2\% dos universitários brasileiros referiram uso do álcool em algum momento da vida ${ }^{17}$. Em Portugal, estudos semelhantes demonstraram resultados ligeiramente menores, com prevalência por volta de 75\%18,19. Em estudos brasileiros realizados com estudantes universitários da área da saúde, em São Paulo, Maceió, Juiz de Fora, Curitiba, Belo Horizonte e Montes Claros, foram identificadas prevalências, respectivamente, de 84,7\%, 90,4\%, 91\%, 90,4\%, $85,3 \%$ e $74,9 \% \%^{2,20-24}$.

A quantidade que define a prática do BPE é de cinco ou mais doses de bebidas alcoólicas em uma única ocasião, independentemente da frequência de consumo. Neste estudo obtivemos um percentual mais elevado, comparado a outros autores que pesquisaram universitários da área da saúde. Na população investigada, a prevalência de BPE foi 
alta $(56,1 \%)$, enquanto no I Levantamento Nacional sobre o Uso de Álcool, Tabaco e Outras Drogas, realizado em 2010, foi de 25,8\%. Em dois estados brasileiros - Minas Gerais e São Paulo -, a prevalência de BPE foi de $27,7 \%$ e $25,6 \%$, respectivamente. Ainda, em estudo recente publicado no Brasil, a prática foi referida por 15,3\% dos universitários. Logo, tornam-se relevantes estudos e medidas preventivas para o cuidado com a saúde dos universitários, pois o consumo de álcool é uma prática relativamente comum que pode causar danos e prejuízos ao indivíduo, além disso, pode prejudicar a vida acadêmica e social desses jovens, já que o uso de álcool pode resultar em posturas inadequadas e na adoção de comportamentos de risco ${ }^{17,21,24,25}$.

$O$ fato de praticar BPE teve significância estatística ( $p<$ $0,050)$ quando relacionado à frequência em festas, pois a maioria dos alunos que praticam BPE frequentam eventos com oferta de bebidas alcoólicas esporadicamente, que podem ser mensais ou quinzenais. Ou seja, o indivíduo não vai com tanta periodicidade a festas ou encontros com amigos, mas, quando vai, consome quantidade elevada de álcool, caracterizando o BPE.

Os dados apresentados revelam que os homens têm uma prática de consumo de álcool mais acentuada em comparação com as mulheres, corroborando resultados encontrados em outros estudos nacionais e internacionais ${ }^{24,26}$. Em estudos realizados no Rio Grande do Sul, Brasil, em universidade pública ${ }^{27,28}$, o fato de ter uma religião, independentemente de qual seja, e ser praticante semanalmente, foi um fator que ocasionou escores no AUDIT e prática de BPE significativamente menores, em comparação com estudantes que têm uma prática eventual ou não seguem com regularidade uma opção religiosa, resultado compatível com os de outros estudos nacionais e internacionais ${ }^{2,20,21,26,29}$.

Em relação à zona de risco em que os estudantes se encontram, segundo o AUDIT, tanto para os 12 cursos no geral como para os cursos analisados separadamente - Farmácia (78,6\%), Medicina (69,4\%) e Enfermagem (75,7\%) -, eles se encontram na zona de baixo risco, semelhante ao estudo realizado em São Paulo ${ }^{29}$.

Percebemos que a população universitária apresenta padrões particulares de uso de álcool e fatores de risco relacionados ao beber problemático, que diferem da população geral, por exemplo, as normas sociais e comportamentais específicas dessa população, o fato de grande parte dela morar longe da família e com outros jovens, distanciando, assim, do possível controle familiar, e ainda a oferta demasiada de festas e locais de lazer com venda livre de bebidas alcoólicas. Nesse contexto, constatamos no estudo que esses fatores interferem no comportamento quanto ao uso de álcool, propiciando maior consumo.

A universidade é uma oportunidade de sair de casa e ter independência e autonomia, o que é legitimado pelas festas e possíveis atitudes inconsequentes. Por outro lado, pode haver sentimentos depressivos e de solidão pela pressão de ter que morar sozinho, longe da família, de atender às expectativas próprias e às dos pais, pela preocupação em terminar o curso e arranjar emprego, pela necessidade de integração no grupo de pares e pelo próprio desafio imposto pela vida acadêmica. Convém ressaltar que essas situações de estresse são devidas à grande cobrança da família e da instituição de ensino, e à competitividade do mercado, exigindo estágios, dedicação e muito estudo, o que pode comprometer gradativamente o tempo de lazer e prejudicar a qualidade de vida do acadêmico. Nesse contexto, o álcool parece ser uma válvula de escape que ajuda a esquecer os problemas ou até mesmo uma estratégia de enfrentamento ${ }^{11,30}$.

O uso abusivo de álcool tem repercussão importante e é um grave problema de saúde pública e educação em saúde, pois esses universitários serão profissionais da saúde e estarão inseridos no mercado de trabalho. São necessárias, então, uma política clara quanto ao uso de drogas e álcool pelos estudantes, informações científicas e educação com treino de habilidades para melhor lidar com estresse, levando os estudantes a assumirem responsabilidade por seus atos e terem consciência dos perigos. Estratégias desse tipo têm se mostrado úteis na prevenção da dependência química e têm sido adotados mundialmente ${ }^{31,32}$.

Há necessidade de as universidades estabelecerem uma política objetiva de orientação sobre o uso de drogas e álcool para os estudantes, incluindo mudanças curriculares e programas de prevenção. Estudos realizados em Portugal e no Brasil mostram evidências da sua eficácia e sugerem a adoção de intervenções breves para auxiliar na redução do consumo de álcool pela população ${ }^{33,34}$, podendo ser uma medida governamental a ser adotada na saúde primária.

Ainda, cabe aos governantes atenção maior a essa população, com punições mais rigorosas e leis mais efetivas. Simplesmente fornecer informação parece não ser suficiente, na medida em que nem sempre o conhecimento sobre as consequências do uso abusivo do álcool é suficiente para ser transformado em práticas seguras ${ }^{29,31,35}$.

A experiência universitária, em muitos casos, dá aos estudantes a primeira oportunidade de ser parte de um grande grupo, sem supervisão familiar. Isso os torna mais vulneráveis a tentar romances e experiências previamente proibidas, e algumas vezes ilícitas. Evidências científicas apontam o álcool como a droga mais utilizada entre os universitários brasileiros e essa utilização é iniciada, em média, a partir de 16 anos. Um estudo realizado em Portugal no ano de 2011 mostrou que o primeiro consumo de álcool na população estudada ocorreu entre 13 e 15 anos de idade, preferencialmente na companhia de amigos, e $43 \%$ foram levados ao consumo por curiosidade ${ }^{2,36}$. 
Além disso, o álcool está relacionado a doenças de alto risco, como patologias cardiovasculares e estomacais, cirrose, hepatite, entre outras, mais prevalentes entre adultos com mais de 25 anos, e o uso é mais frequente entre bebedores jovens. Esse consumo está associado a várias consequências negativas e é uma das causas principais de morbidade e mortalidade entre os universitários. A literatura tem destacado, ainda, a associação de fatores ambientais, tais como religiosidade, condição de trabalho e uso de substâncias entre os amigos e parentes, e de fatores psicossociais, como conflitos com pais e sentimentos negativos e de solidão, com o uso de álcool e tabaco entre os estudantes $20,35,37,38$.

Nessa perspectiva, a monitorização do BPE e do abuso e dependência de bebidas alcoólicas, por meio de dados epidemiológicos no ambiente universitário, torna-se uma prática necessária de vigilância em saúde, não apenas por se tratar de futuros profissionais da área da saúde, mas para o desenvolvimento de políticas públicas e estratégias de prevenção que visem diminuir o abuso e a dependência. Além disso, a verificação do impacto na saúde e na economia provocado pelo consumo abusivo do álcool pode nortear políticas públicas e intervenções por meio da instituição de ensino $2,5,38$.

Por se tratar de estudo descritivo e transversal, não possibilita a compreensão das causas dos desfechos observados em relação ao consumo de álcool entre os universitários da área da saúde. Assim, para o discernimento de tais comportamentos, bem como a definição de estratégias para intervir no perfil e práticas de consumo de bebidas alcoólicas, são necessários estudos adicionais com delineamentos apropriados.

\section{CONCLUSÃO}

O consumo de álcool entre os universitários avaliados é elevado e semelhante a outras evidências nacionais e internacionais.

A frequência do BPE foi quase o dobro quando comparada a de outros estudos.

O perfil do consumo foi predominantemente de baixo risco, demonstrando a exposição precoce ao álcool para os futuros profissionais de saúde.

As características que mais se associaram ao consumo de álcool foram ser solteiro, do sexo masculino e frequentar festas semanalmente. A prática religiosa semanal mostrou-se como um fator protetor em relação ao consumo de álcool.

Desse modo, ficou evidente a necessidade de ações educativas para a promoção da saúde do estudante universitário, inclusive prevenindo o consumo de álcool e outras substâncias, para que, além de cuidar da sua saúde, os sujeitos tenham conhecimentos necessários para informar, educar e promover a saúde da população.

\section{CONTRIBUIÇÕES INDIVIDUAIS}

Todos os autores contribuíram significativamente na concepção e desenho do estudo, análise, interpretação dos dados, bem como na elaboração do artigo, com a revisão crítica de seu conteúdo intelectual, assim, aprovando a sua versão final a ser publicada.

\section{CONFLITOS DE INTERESSE}

Os autores Marina Pelicioli, Cristiane Barelli, Carla Beatrice Crivellaro Gonçalves, Siomara Regina Hahn e José Ivo Scherer declaram que não houve conflito de interesse na realização deste trabalho. Esta pesquisa integrou o trabalho de conclusão do curso de graduação em Farmácia da autora principal.

\section{AGRADECIMENTOS}

Agradecemos a todos os estudantes que participaram da pesquisa, por sua disponibilidade e colaboração com o estudo, e também à Universidade de Passo Fundo, que apoiou e autorizou a realização deste trabalho. Sendo assim, declaramos que este trabalho não recebeu qualquer contribuição, subsídio ou bolsa e que foi financiado com recursos próprios dos pesquisadores.

\section{REFERÊNCIAS}

1. Fontana AM. Manual de clínica em psiquiatria. São Paulo: Atheneu; 2005. 511p.

2. Silva LVER, Malbergier A, Stempliuk VA, Andrade AG. Fatores associados ao consumo de álcool e drogas entre estudantes universitários. Rev Saúde Pública. 2006;40(2):280-8.

3. Carlini-Cotrim B, Gazal-Carvalho C, Gouveia N. Comportamento de saúde entre jovens estudantes das redes pública e privada da área metropolitana do estado de São Paulo. Rev Saúde Pública. 2000;34(6):636-45.

4. Andrade AG, Anthony JC, Silveira CM. Álcool e suas consequências: uma abordagem multiconceitual. São Paulo: Manole; 2009. 208p.

5. Duailibi $S$, Laranjeira R. Políticas públicas relacionadas às bebidas alcoólicas. Rev Saúde Pública. 2007:41(5):839-48.

6. Anderson P, Baumberg B. Alcohol in Europe: a public health perspective. A report for the European Commission. London: Institute of Alcohol Studies; 2006.

7. Nunes JM, Campolina LR, Vieira MA, Caldeira A. Consumo de bebidas alcoólicas e prática do binge drinking entre acadêmicos da área da saúde. Rev Psiquiatr Clín. 2012;39(3).

8. National Institute on Alcohol and Alcoholism - NIAAA. Helping patients who drink too much: a clinician's guide, National Institute on Alcohol and Alcoholism. 2005. Disponível em: https://pubs.niaaa.nih.gov/publications/practitioner/cliniciansguide2005/guide.pdf. Acesso em: 10 ago 2017.

9. Shakeshaft AP, Bowman JA, Sanson-Fisher RW. Comparison of three methods to assess binge consumption: One-Week Retrospective Drinking Diary, AUDIT, and Quantity/Frequency. Subst Abus. 1998;19(4):191-203.

10. Brasil. Ministério da Saúde. Conasems. O SuS de A a Z. za ed. Brasília: Ministério da Saúde; 2009. Disponível em: http://portalarquivos.saude.gov.br/images/pdf/2013/agosto/28/ sus-3edica0-completo-190911.pdf. Acesso em: 22 dez. 2015. 
11. Silveira C, Norton A, Brandão I, Roma-Torres A. Saúde mental em estudantes do ensino superior: experiência da consulta de Psiquiatria do Centro Hospitalar São João. Acta Med Port. 2011;24(S2):247-56.

12. Luis MAV, Pillon SC. 0 conhecimento dos alunos de enfermagem sobre álcool edrogas. Rev Eletrônica Enfermagem. 2003;5(1):21-7.

13. Cochran W. Sampling techniques. 3rd ed. New York: John Wiley \& Sons; 1986.

14 Jomar RT, Paixão LAR, Abreu AMM. Alcohol Use Disorders Identification Test (AUDIT) e sua aplicabilidade na atenção primária à saúde. Rev APS. 2012;15(1):113-7.

15. Aliane PP, Lourenço LM, Ronzani TM. Estudo comparativo das habilidades sociais de dependentes e não dependentes de álcool. Psicol Estud. 2006;11(1):83-8.

16. Moretti-Pires RO, Corradi-Webster CM. Adaptação e validação do Alcohol Use Disorder Identification Test (AUDIT) para população ribeirinha do interior da Amazônia, Brasil. Cad Saúde Pública. 2011;27(3):497-509.

17. Brasil. Presidência da República. Secretaria Nacional de Políticas sobre Drogas. I Levantamento Nacional sobre o Uso de Álcool, Tabaco e Outras Drogas entre Universitários das 27 Capitais Brasileiras/Secretaria Nacional de Políticas sobre Drogas; GREA/IPQ-HCFMUSP. Org.: Andrade AG, Duarte PCAV, Oliveira LG. Brasilia: SENAD; 2010.

18. Grácio JCG. Determinantes do consumo de bebidas alcoólicas nos estudantes do ensino superior de Coimbra [e-book]. Universidade de Coimbra; 2009 Disponível em: https://estudogeral.sib.uc.pt/handle/10316/13509. Acesso em: 20 dez. 2015.

19. Cabral LR. Consumo de bebidas alcoólicas em rituais/praxes académicas [e-book]. Porto: Universidade do Porto; 2007. Disponível em: https://repositorio-aberto.up.pt/ bitstream/10216/7207/2/Doutoramento\%20Lidia\%20d0\%20Rosrio\%20Cabral\%20 Agosto2007.pdf. Acesso em: 9 jan. 2016.

20. Pedrosa AAS, Camacho LAB, Passos SRL, Oliveira RVC. Consumo de álcool entre estudantes universitários. Cad Saúde Pública. 2011;27(8):1611-21.

21. Carneiro EB, Braga RT, Silva LFD, Nogueira MC. Fatores associados a beber pesado episódico entre estudantes de medicina. RBEM. 2012;36(4):524-30.

22. Chiapetti N, Serbena CA. Uso de álcool, tabaco e drogas por estudantes da área de saúde de uma universidade de Curitiba. Psicol Reflex Crit. 2007;20(2):303-13.

23. Amorim AVC, Kikko EO, Abrantes MM, Andrade VLA. Álcool e alcoolismo: estudo de prevalência entre discentes do curso de Medicina da UNIFENAS em Belo Horizonte - Minas Gerais. RMMG. 2008;18(1):1623.
24. Cardoso FM, Barbosa HA, Costa FM, Vieira MA, Caldeira AP. Fatores associados à prática do binge drinking entre estudantes da área da saúde. Rev CEFAC. 2015;17(2):475-84.

25. Reis A, Barros J, Fonseca C, Parreira L, Gomes M, Figueiredo I, et al. Prevalência da ingestão de álcool nos adolescentes - Estudo PINGA. Rev Port Clin Geral. 2011;27(4):338-46.

26. Instituto Nacional de Estatística \& Instituto Nacional de Saúde Dr. Ricardo Jorge (2009). Inquérito Nacional de Saúde (40) 2005/2006. Lisboa: INE, I.P. e INSA, I.P.

27. Pillon SC, Corradi-Webster CM. Teste de identificação de problemas relacionados ao uso de álcool entre estudantes universitários. Rev Enferm UERJ. 2006;14(3):325-32.

28. Peuker AC, Fogaça J, Bizarro L. Expectativas e beber problemático entre universitários. Psic Teor Pesq. 2006;22(2):193-200.

29. Silva EC, Tucci AM. Estudo transversal sobre o uso de risco de álcool em uma amostra de estudantes de uma universidade federal brasileira. J Bras Psiquiatr. 2014;63(4):317-25.

30. Teixeira MAP, Dias AGG, Wottrich SH, Oliveira AM. Adaptação à universidade em jovens calouros. Psicol Esc Educ. 2008;12(1):185-202.

31. Kerr-Correa F, Andrade AG, Bassit AZ, Boccuto NMVF. Uso de álcool e drogas por estudantes de medicina da Unesp. Rev Bras Psiquiatr. 1999;21(2):95-100.

32. Boruchovitch E. Fatores associados a não utilização de anticoncepcionais na adolescência. Rev Saúde Pública. 1992;26(6):437-43.

33. Ribeiro C. Medicina geral e familiar e a abordagem do consumo de álcool: detecção e intervenções breves no âmbito dos cuidados de saúde primários. Acta Med Port. 2011;24(S2):355-68.

34. Marques ACPR, Furtado EF. Intervenções breves para problemas relacionados ao álcool. Rev Bras Psiquiatria 2004;26(Supl 1):28-32

35. Barbosa Filho VC, Campos W, Lopes AS. Prevalence of alcohol and tobacco use among Brazilian adolescents : a systematic review. Rev Saúde Pública. 2012;46(5):901-17.

36. Marques M, Viveiro C, Passadouro R. Uma velha questão numa população jovem: 0 consumo do álcool nos adolescentes escolarizados. Acta Med Port. 2013; 26(2):133-8.

37. Wannmacher L. Interações de medicamentos com álcool: verdades e mitos. Braśilia: Organização Pan-Americana da Saúde/Organização Mundial da Saúde; 2007. v. 4, n. 12, p. 1-6.

38. Wagner GA, Oliveira LG, Barroso LP, Nishimura R, Ishihara LM, Stempliuk VA, et al. Drug use in college students : a 13-year trend. Rev Saúde Pública. 2012;46(3):497-504. 